\title{
Formative Evaluation of the Families SHARE Disease Risk Tool among Low-Income African Americans
}

\author{
Kayla de la Haye $^{\mathrm{a}}$ Calandra Whitted $^{\mathrm{b}, \mathrm{c}}$ Laura M. Koehly \\ aDepartment of Preventive Medicine, Keck School of Medicine, University of Southern California, Los Angeles, CA,

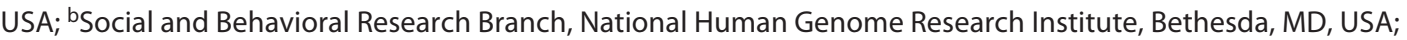 \\ 'Behavioral Sciences and Health Education, Florida A\&M University, Tallahassee, FL, USA
}

\section{Keywords \\ Family health history - African American - Evaluation • Educational tool · Families SHARE · Intervention · Mixed methods}

\begin{abstract}
Introduction: Family Health Histories $(\mathrm{FHH})$ have been endorsed by the surgeon general as a powerful yet underutilized tool for identifying individuals at risk for complex chronic diseases such as diabetes, heart disease, and cancer. $\mathrm{FHH}$ tools provide a mechanism for increasing communication about disease history and motivating behavior change to reduce disease risk. A critical gap in translation efforts includes a lack of research that adapts and evaluates tools for low-income, minority populations who experience disparities in chronic disease. Methods: This study is a formative mixed-methods evaluation of an evidence-based $\mathrm{FHH}$ intervention called "Families SHARE" among African Americans residing in low-income neighborhoods. Participants $(N=51)$ completed assessments before and 6 weeks after receiving the intervention, including surveys and focus groups. We evaluated (a) their use, understanding, and perceived value of the tool; (b) if the intervention led to increased intentions
\end{abstract}

to adopt disease risk-reducing behaviors among those with heightened disease risk, given their $\mathrm{FHH}$; and (c) acceptability of and recommendations for the tool. Results: The quantitative and qualitative data indicated that this population valued and used the tool, and it prompted communication about FHH with family, friends, and others. Receipt of the intervention resulted in mixed accuracy of their perceived disease risk, and it did not shift intentions to change health behaviors. Qualitative data provide insights for future iterations of the Families SHARE tool. Conclusion: Families SHARE is an engaging $\mathrm{FHH}$ tool that can be further tailored to optimize its value and benefits for low-income African Americans.

This is a work of the U.S. Government and is not subject to copyright protection in the United States. Foreign copyrights may apply. Published by S. Karger AG, Basel.

\section{Introduction}

Heart disease, diabetes, and cancer are etiologically complex with multiple risk factors (e.g., environment, genetic, and lifestyle), which tend to cluster in families, resulting in a patterning of disease among biologically related family members. Family Health History (FHH) is a karger@karger.com www.karger.com/phg

Karger $\stackrel{\text { ' }}{5}$

BOPEN ACCESS
Published by S. Karger AG, Basel.

This is an Open Access article licensed under the Creative Common Attribution-NonCommercial-4.0 International License (CC BY-NC) (http://www.karger.com/Services/OpenAccessLicense), applicable to the online version of the article only. Usage and distribution for commercial purposes requires written permission.
Correspondence to:

Kayla de la Haye, delahaye@usc.edu 
genomic tool detailing disease diagnoses within the family to aid in the interpretation of disease risk [1]. FHH is used in clinical settings to assess individuals' disease risk and to personalize recommendations for preventive health behaviors, including clinical screenings beginning at an earlier age. For FHH-based risk assessment to be effective, patients must bring to the clinical encounter accurate information about family members' disease diagnoses. FHH can also be an effective intervention tool in nonclinical family settings. Introduction of FHH information within families has been shown to activate conversations about family risk, resulting in improved knowledge of FHH [2]. Moreover, families are an important social context for intervention and lifestyle-focused disease prevention because members tend to share behavioral, social, economic, and environmental risk factors and members can influence one another's potential for behavior change [3]. Thus, FHH is a powerful and underutilized tool that helps individuals and families understand their risk for diseases with genetic underpinnings and encourage the adoption of risk-reducing behaviors [4].

Initiatives to accelerate the use and impact of $\mathrm{FHH}$ tools within families and communities involve the analysis of information on family morbidity from heritable diseases such as diabetes, heart disease, or cancer; feedback on individuals' disease risk based on FHH trees or pedigrees; and feedback on behavioral strategies for risk modification [3, 5-7]. These programs have demonstrated efficacy in prompting discussion about family disease history and motivating behavior change to reduce disease risk $[2,6,8,9]$. A critical gap in translation efforts is a lack of research that adapts and evaluates tools for low-income African American families, accounting for the family, social, and environmental contexts in which they are embedded [7, 10]. Tailoring FHH tools for African American families - based on the unique structure and composition of family systems [11], the roles of family members as a source of FHH information, and given the impact of structural racism in medicine and health care on medical mistrust [12] - may increase the tool's acceptability and impact. This project addresses the gap by evaluating the acceptability and usability of an evidence-based FHH tool for use in community settings in an under-resourced, African American community.

\section{Families SHARE}

Families SHARE (Sharing Health Assessment and Risk Evaluation) was developed by the National Human Genome Research Institute (NHGRI) and designed to ed-

Evaluation of Families SHARE for African Americans ucate individuals on FHH-based risk of heart disease, diabetes, breast cancer, and colorectal cancer [13]. Like other FHH tools, Families SHARE involves the analysis of information on family morbidity from diseases, feedback about disease risk, and information on behavioral strategies for risk modification. Families SHARE is unique because it adopts 2 innovative approaches to implementation. The first is a social network and a communal coping perspective, promoting increased communication about disease history in the family that helps motivate collective behavior change $[14,15]$. The second is a train-the-trainer framework, where family health educators are given the skills to evaluate their own disease risk and their family members' risk. This contrasts with $\mathrm{FHH}$ initiatives that adopt an individualistic approach, where FHHs are recorded, "experts" or computer software processes the information, and the results of disease risk assessment are provided to individuals.

Tailoring Families SHARE for Populations

Experiencing Health Disparities

African Americans experience a disproportionate burden of chronic disease compared to other race and ethnic groups in the USA and face many social and structural barriers to adopting disease risk-modifying behaviors such as increased screening or adopting a healthy diet. Disparities persist for African Americans for diabetes, heart disease, and cancer [16, 17]. For example, $17 \%$ of African American adults have diabetes, the highest of any US racial/ethnic population [18]. The prevalence of obesity is significantly higher among non-Hispanic Black women than among non-Hispanic white women (56 and $39 \%$, respectively) [19]. Diabetes and obesity are risk factors for cardiovascular disease, and the overall death rate attributed to cardiovascular disease is higher among nonHispanic Blacks (352.4 per 100,000 for male individuals and 248.6 per 100,000 for female individuals) than among non-Hispanic whites (271.9 per 100,000 for males and 188.1 per 100,000 for females) [20]. African American families with low socioeconomic status have the greatest exposure to social and ecological health risk factors that contribute to higher rates of diabetes and heart disease, and poorer disease outcomes, representing an important population to focus community programs aimed at risk reduction.

Family structures among African Americans are an important consideration for tailoring the Families SHARE workbook. About half (54\%) of African American children are living in single-parent households [21]. African Americans have greater connections to "social kin" - that 
is, kin who are culturally defined, instead of defined based on genetic relatedness - who may be important sources of information and support for health and well-being [11, 22]. Structural features of these family systems may be important factors in communication and knowledge of $\mathrm{FHH}$, and influence family members' roles in response to disease risk and adoption of risk-reducing behaviors.

\section{Evaluation of FHH Interventions among Low-Income African Americans}

This study seeks to inform the scale-up of a family and community-centered $\mathrm{FHH}$ program that engages low-income African Americans to utilize FHH to improve their health. The potential for using FHH initiatives among African American populations was suggested by the Healthy Black Family Project, a community-based intervention targeting health disparities among African Americans $[23,24]$. A component of this project assessed FHH and was found to be effective in promoting the adoption of accurate disease risk perceptions among African Americans [25], suggesting that $\mathrm{FHH}$ tools have the potential to motivate healthy behavior change.

Qualitative and mixed-methods research has found that compared to white Americans, African Americans may communicate less with their family members about FHH $[26,27]$ and that diverse patterns of communication about FHH within African American families are impacted by family member characteristics and relationship quality $[28,29]$. Overall, more research is needed to understand the acceptability and effectiveness of FHH to change behavior among low-income, African American families and to understand how interventions can be tailored to family structures and systems.

\section{Current Study}

The goal of the current study is to conduct a formative evaluation of Families SHARE to tailor the tool and approach to be administered in low-income, African American communities. Findings are based on data collected from a sample of African American adults living in 2 lowincome neighborhoods in Baltimore, $\mathrm{MD}$, and Washington, DC. Assessments were conducted at baseline and approximately 6 weeks after receipt of the workbook. Focus groups were conducted to gather richer qualitative data. The aims were to assess (a) if this population utilized, understood, and valued the tool; (b) the effectiveness of the tool in eliciting change in intentions to adopt risk-reducing behaviors among participants with increased disease risk; and (c) participant feedback on the acceptability of the tool and their recommendations for improvement.

\section{Materials and Methods}

\section{Procedure and Sample}

Community outreach was used to recruit participants from 2 neighborhoods in Baltimore, $\mathrm{MD}$, and Washington, DC, where the majority were low-income households and $>90 \%$ residents were identified as African American. Interested individuals were contacted by phone and provided information about the study and to determine eligibility: (1) able to read and speak English, (2) currently live in one of the selected neighborhoods, (3) 18-70 years old, (4) have at least one biological child, (5) identify as Black/African American, and (6) not adopted. Informed consent was obtained from eligible participants by phone.

Consented participants $(N=62)$ were contacted by research assistants for baseline assessments. Within 1 week of assessment, participants were mailed their personalized Families SHARE workbook. Participants were recontacted within 6 weeks of receiving their workbook to complete the follow-up survey. Upon completion of all follow-up assessments, 10 study participants took part in 2 focus groups. The study protocol was approved by the Institutional Review Board of the National Human Genome Research Institute (NHGRI: 12-HG-0023).

\section{Characteristics of the Analytic Sample}

Of the 62 participants who were consented to the study, 51 completed baseline and follow-up assessments. Participants' ages ranged from 30 to $72(\mathrm{M}=53.5, \mathrm{SD}=10.2)$ years, and $74.5 \%$ were female (38 of 51). All participants (100\%) identified as Black or African American and were born in the USA. Other relevant demographic and health characteristics are described in the Results.

\section{Families SHARE Workbook}

Each participant received a package that included a cover letter, a Families SHARE workbook, and a set of magnets to remind participants to make healthy lifestyle choices and to update their FHH. The Families SHARE workbook focuses on FHH-based risk of heart disease, diabetes, breast cancer, and colon cancer and comprises the following components: (1) a graphical sample $\mathrm{FHH}$ pedigree; (2) participants' personalized $\mathrm{FHH}$ pedigree detailing their family history of the 4 target diseases, based on information they provided at baseline; (3) disease factsheets defining each disease and associated risk factors, (4) a risk worksheet containing an algorithm for assessing increased disease risk based on FHH (the algorithm was based on a review of the literature connecting $\mathrm{FHH}$ to disease outcomes, as well as clinical recommendations), and (5) healthy lifestyle and health screening recommendations for risk reduction and early detection tailored to an "average" or "increased" disease risk level (for examples of the Families SHARE material see [13]).

\section{Measures}

Baseline and follow-up surveys were administered by telephone. Baseline surveys gathered information about participant demographics, FHH, and health status. Follow-up surveys assessed health status as well as acceptability and usability of the Families SHARE workbook. 


\section{Key Outcomes}

Utilization of the Families SHARE Workbook: This was assessed by asking participants if by using the workbook they could (1) assess their own degree of risk for each disease and (2) assess their family member's degree of risk for each disease (yes/no). Participants reported on who they talked to in their family to get $\mathrm{FHH}$ information, who they shared their Families SHARE information with, and if they intended to share this information with anyone else in future. They also reported on who (if anyone) they physically showed the Families SHARE workbook to and if those they shared the workbook with could assess their own degree of risk.

Participant's Understanding of the Families SHARE Workbook: This was evaluated based on self-reported understanding of the entire workbook and their understanding of each section: the cover letter, the sample pedigree, their personalized pedigree, the disease risk worksheet, and the healthy recommendations $(1=I$ did not understand at all, $7=I$ completely understood). Participants indicated if after using the workbook, they could identify ways they and their family could reduce their risk for disease (yes/no).

Risk Perceptions: Understanding of the workbook material was also evaluated by examining the accuracy of participants' risk perceptions (i.e., increased risk vs. average risk) for the 4 focal diseases at follow-up. Participants who indicated they were able to use the algorithm provided their risk assessment (perceived risk; PR) for each disease as measured by the question "Are you at increased risk for colorectal cancer/breast cancer/heart disease/type 2 diabetes" ( 1 = yes, 2 =no, 3 =don't know). This was compared to their risk for each disease ( 1 = average risk, 2 = increased risk), derived from an analysis of their self-reported $\mathrm{FHH}$ using the same risk algorithm (Families SHARE risk [FSR]). This information was used to categorize participants into the following disease-specific risk categories: (1) diagnosed by a physician; (2) not diagnosed, with increased FSR and accurate increased PR; (3) not diagnosed, with average FSR and accurate average PR; (4) not diagnosed, with increased FSR but inaccurate average PR; and (5) not diagnosed, with average FSR but inaccurate increased PR.

Health Behavior Change: Baseline and follow-up surveys measured intention and confidence to change several target health behaviors: limit alcohol consumption, increase fruit and vegetable consumption, increase fiber consumption, stop smoking, and increase physical activity. Participants indicated whether they intended to change each behavior $(1=y e s, 0=n o)$, and if so, how confident they were that they could make each change within the next year $(1=$ not at all confident, $7=$ very confident $)$. A summary measure for intention/confidence to change each behavior was computed by multiplying the intention and confidence scores, resulting in an overall scale where 0 denotes no intention to change the behavior, 1 intend to change the behavior, but not at all confident, up to 7 denotes intend to change the behavior and very confident.

Recommendations for Improvement: Participant recommendations to improve Families SHARE overall and each of the key workbook components were assessed by open-ended questions in the survey and during the focus groups.

Personal and Family Health History: At baseline, participants indicated if a health professional had ever diagnosed them with colorectal cancer, breast cancer, type 2 diabetes, or heart disease, and the age of diagnosis for each disease. Participants were prompted about all first- and second-degree biological relatives (mother, father, maternal and paternal grandmother and grandfather, mothers' siblings, fathers' siblings, siblings, siblings' children, and their children) and whether or not each individual had been diagnosed with any of the 4 focal diseases and the age of diagnosis. This information was entered into Progeny software [30] to generate and draw a personalized $\mathrm{FHH}$ pedigree that was later provided to participants within the Families SHARE workbook.

\section{Health Status}

Weight Status: Participant's self-reported height and weight were used to calculate their body mass index $\left(B M I ; B M I=\mathrm{kg} / \mathrm{m}^{2}\right)$. The Centers for Disease Control (CDC) guidelines were used to categorize BMI scores into the following weight categories: underweight $(<18.5)$, normal weight $(18.5$ to $<25)$, overweight $(25$ to $<30)$, obese class $1(30$ to $<55)$, obese class $2(35$ to $<40)$, and obese class 3 (40 or higher).

Self-Rated Health: This was assessed by the question "would you say your health is..."? with 5 response options ranging from $1=$ excellent and $5=$ poor.

Health Behaviors: Smoking behavior was assessed by asking participants "Do you smoke tobacco, including cigarettes, a pipe, or cigars"? where 1 = yes; $2=$ no, I used to smoke; $3=$ no, I have never smoked.

Alcohol consumption: It was measured by asking participants how many alcoholic beverages (standard servings) they drank in the previous 7 days.

Dietary intake: This was assessed by participant reports of how many cups of fruit and how many cups of vegetables they typically eat each day (examples of one cup of fruit and one cup of vegetables were provided).

Physical activity: This was assessed by asking participants about their engagement in vigorous activities, moderate activities, and walking, in the previous 7 days. For each of the 3 types of physical activity, they were asked to (1) report how many days per week (in the past 7 days) they did activity for at least $10 \mathrm{~min}$ at a time (examples of each activity type were provided), and if they reported 1 or more days they were asked (2) to report on how much time (in hours and minutes) they usually spent on one of those days doing the activity. These data were used to compute their typical time (in minutes) spent doing each activity in the past week.

Health-Care Insurance: This was assessed by the question "Do you currently have health-care coverage, such as insurance, Medicare, or medical assistance"? (yes/no)

\section{Demographics}

Baseline surveys measured demographic characteristics including gender, age, marital status, race/ethnicity, highest level of formal education, household income, and household composition.

\section{Analytic Strategy}

FHH Risk Algorithm

For each disease, an indicator of increased risk was computed using the algorithm provided in the Families SHARE workbook. Specifically, participants were at increased risk for a given disease (diabetes, heart disease, and colon cancer) if they had a first-degree relative diagnosed with the condition or 2 second-degree relatives diagnosed. A similar algorithm was used for breast cancer in the case of female participants; if a female family member had a breast cancer diagnosis, both female and male participants were at increased risk. 
Table 1. Descriptive statistics for participant demographics $(N=$ 51)

\begin{tabular}{lc}
\hline Characteristic & Statistic \\
\hline Highest level of education, \% & \\
Less than high school & 13.7 \\
High school diploma/GED & 31.4 \\
$\quad$ Some college/associate degree/ & \\
$\quad$ technical or vocational school & 39.2 \\
Bachelor degree or more & 15.7 \\
Yearly household income, pre-tax, \% & \\
20,000 or less & 43.1 \\
20,001-35,000 & 25.5 \\
35,001-50,000 & 7.8 \\
50,001-75,000 & 11.8 \\
75,001 or higher & 3.9 \\
Not reported & 7.8 \\
Marital status, \% & \\
Single & 56.9 \\
Married/living as married & 19.6 \\
Separated & 2.0 \\
Divorced & 13.7 \\
Widowed & 7.8 \\
Number of children & \\
M (SD) & $2.55(1.43)$ \\
Min, max & 1,7 \\
Number of people living in their residence (excluding \\
participant) \\
M (SD) \\
Min, max & $1.75(1.32)$ \\
\hline
\end{tabular}

Survey Data

Survey data were analyzed using R and SPSS 22.0. Descriptive measures were computed to describe sample characteristics, utilization and understanding of the Families SHARE workbook, and behavioral intentions/confidence at baseline and follow-up. Paired $t$ tests were conducted to evaluate differences in behavioral intentions/confidence between baseline and follow-up assessments.

Focus Group Data

Transcripts of the focus groups were reviewed and key themes extracted to summarize qualitative data regarding the Families SHARE workbook and recommendations for improvements.

\section{Results}

\section{Sample Demographics and Health Characteristics}

Demographic characteristics are summarized in Table 1. Few participants (15.7\%) had completed education at a bachelor's level or higher, and the majority of participants lived in households with a yearly income of $\$ 35,000$ or less. The majority of participants were single (56.9\%), they had an average of $2-3$ children, and all lived in households with at least one $(\max =5)$ other individual.

A range of health characteristics and behaviors are summarized in Table 2. Diabetes was the most prevalent disease diagnosed in the sample (21.6\%), while 2 participants had been diagnosed with heart disease, 2 with breast cancer, and none with colorectal cancer. Rates of overweight and obesity were high $(86.3 \%)$, and a third of the sample rated their health as poor or fair. About one-third of the sample contained current smokers, and $45 \%$ had consumed alcohol in the past week ( $N=5$ had consumed alcohol in excess of recommended limits); $39.2 \%$ of participants did not meet national physical activity guidelines of 5 or more days/week with $30+$ minutes of moderate or vigorous physical activity. The average consumption of fruits and vegetables was below the recommended USDA guidelines for adults (between 1.5 and 2 cups/day fruit, and between 2 and 3 cups/day of vegetables, depending on gender and age).

\section{Utilization of the Families SHARE Workbook}

All but one (50 of the 51) participants (98.0\%) reported that they could assess their own disease risk using the workbook. Of these 50 participants, 32 (64\%) tried to use the workbook to assess other family members' risk, and almost all did this successfully: 25 of these 32 said "yes," they did this successfully, and 5 said they were able to assess most or some of their family members' risk.

Participants who used the workbook $(n=50)$ engaged with other family members and people in their social networks about the Families SHARE workbook. Almost half of these participants $(48 \%, 24$ of 50$)$ reported that they talked to people in their family to get information on their FHH. The workbook feedback was shared with others: $76 \%$ of participants ( 38 of 50 ) talked to others about the feedback and $70 \%$ (35 of 50) said they showed the workbook to someone else (including family, friends, work colleagues, and physicians), with under half ( $43 \%, 15$ of the 35) indicating that the people they showed it to tried to assess their own risk. Three-quarters of participants $(74 \%, 37$ of 50$)$ said that they intended to share the workbook with other people in future, including family members, friends, pastors, and health-care providers.

The focus group (FG) data reinforced these findings, with participants reporting that the workbook was useful for sharing with family members, social kin, and healthcare providers:

"I showed her [daughter] this just today. So, she wants to look at it and read through it. Maybe it will wake her up a little bit on her intake." (female, FG1) 
Table 2. Descriptive statistics for baseline health characteristics $(N=51)$

\begin{tabular}{|c|c|}
\hline Characteristic & Statistic \\
\hline \multicolumn{2}{|l|}{ Baseline disease diagnoses, $\%$} \\
\hline Diabetes & 21.6 \\
\hline Heart disease & 3.9 \\
\hline Colorectal cancer & 0.0 \\
\hline Breast cancer & 3.9 \\
\hline \multicolumn{2}{|l|}{ Weight category, \% } \\
\hline Non-overweight & 13.7 \\
\hline Overweight & 23.5 \\
\hline Obese Class I & 37.3 \\
\hline Obese Class II & 7.8 \\
\hline Obese Class III & 17.6 \\
\hline \multicolumn{2}{|l|}{ Self-rated health, $\%$} \\
\hline Very good/excellent & 31.4 \\
\hline Good & 35.3 \\
\hline Poor/fair & 33.3 \\
\hline \multicolumn{2}{|l|}{ Smoking behavior, \% } \\
\hline Current smoker & 33.3 \\
\hline Used to smoke & 27.5 \\
\hline Never smoked & 39.2 \\
\hline \multicolumn{2}{|l|}{ Alcohol consumption in the past week } \\
\hline M (SD) standard drinks consumed & $4.5(11.0)$ \\
\hline No standard drinks, $\%$ & 55.0 \\
\hline \multicolumn{2}{|l|}{ Physical activity (PA) } \\
\hline $\mathrm{M}(\mathrm{SD}) \mathrm{min}$ of vigorous PA in the past week & $260.5(593.0)$ \\
\hline $\mathrm{M}(\mathrm{SD})$ min of moderate PA in the past week & $451.3(655.3)$ \\
\hline $\mathrm{M}(\mathrm{SD})$ min of walking in the past week & $388.5(706.1)$ \\
\hline \multicolumn{2}{|l|}{ Dietary intake } \\
\hline M (SD) cups of fruit/day & $1.2(1.1)$ \\
\hline $\mathrm{M}(\mathrm{SD})$ cups of vegetable/day & $1.6(1.0)$ \\
\hline Have health-care coverage/insurance, $\%$ & 96.1 \\
\hline
\end{tabular}

“... I just made it a project. When I was with my girlfriend or my goddaughter, I would talk to her. ...'Let's talk about this. You know I had this ailment, this ailment. So, what do you have"'? (male, FG2)

A common theme in conversations was that the workbook gave participants a sense of ownership over their FHH and that they felt empowered from using and sharing this information with health-care providers:

"I think you go to the doctor and you present this to her, and you say, 'Dr. Smith, this is what I've been working on.' This lets them know that you are concerned and serious about your conditions." (male, FG2)

"After I got it, I think maybe like a month later I had a doctor's appointment, so I took this to the doctor with me... Talked about the heart part." (female, FG1)

"This is mine, I present this. That is real important and the fact that I control this..." (male, FG2)

Evaluation of Families SHARE for African Americans
"I like to have the extra information written down when I go do see a professional. Sometimes I can forget stuff. This is an excellent tool and this tool is more elaborate than any family history they've ever asked me. This gives me some ammunition to present." (male, FG2)

"I guess down through the years hearing tidbits here and tidbits there it was just like when I saw it on paper I was like, 'Now how can I use this for my family"' (female, FG2)

Conversations during the focus groups indicated that the workbooks prompted participants to reach out to family members to gather additional information about their FHH. Some identified "family historians" and older family members as important information sources and often these were women (e.g., mothers, grandmothers, and sisters):

"...my mom, because my mom knows it, she knows it all. She got it. She was the baby, but she knows all of them. She knows all what the brothers had and everything" (female, FG1)

"I would go to my sister when I couldn't figure it out. My sister is the gatekeeper. I would go to her and try to get this information and a lot of it she had. It helped me fill in those blanks." (male, FG2)

One participant identified a male cousin as the "family historian" and source of family health information:

"My cousin in our family calls himself a historian. He has traced our family roots back about seven or eight generations. He was able to tell me about my grandfather on my mother's side." (female, FG2)

One barrier to using the workbook that was raised in the focus groups was having an absentee parent, which participants described as a challenge to getting family health information. As a couple of participants stated,

"I'm just looking at this, and I noticed that I do not have any information about my father, because he was like absentee father all my life. So, I don't know what he died from, and I don't even know his part." (female, FG1)

"if it started with my father... I couldn't do it because I didn't even have the information that I needed to." (female, FG1)

Participants reported that they did not share the Families SHARE workbook with some family members because they didn't think of it, didn't have time, or hadn't seen them/hadn't had the chance over the 6 weeks since they'd received the workbook. Some key reasons that were cited for not intending to share the Families SHARE information with others (26\%) in the future were because they "wouldn't care" or have an interest in this information because "it's just not something they talk about," "they live different lifestyles," or because others "aren't easy to talk to." Participants indicated that they didn't interact with or have close relationships with some family mem-

Public Health Genomics 2021;24:280-290 
Table 3. Summary of disease risk characteristics $(N=51)$

\begin{tabular}{lcccc}
\hline Disease risk characteristic & Diabetes & $\begin{array}{l}\text { Heart } \\
\text { disease }\end{array}$ & $\begin{array}{l}\text { Colorectal } \\
\text { cancer }\end{array}$ & $\begin{array}{l}\text { Breast cancer } \\
\text { (females only, N=38) }\end{array}$ \\
\hline Diagnosed, \% & 21.6 & 3.9 & 0.0 & 5.3 \\
ND with increased FSR, \% & 82.5 & 89.8 & 5.9 & 22.2 \\
ND with increased PR, \% & 40.0 & 38.8 & 5.9 & 5.6 \\
Accuracy of risk perception among ND, \% & & & & \\
$\quad$ Increased FSR and PR (accurate) & 35.0 & 36.7 & 0.0 & 0.0 \\
$\quad$ Average FSR and PR (accurate) & 12.5 & 6.1 & 82.4 & 66.7 \\
$\quad$ Increased FSR but average PR (inaccurate) & 42.5 & 51.0 & 5.9 & 22.2 \\
$\quad$ Increased PR but average FSR (inaccurate) & 5.0 & 2.0 & 5.9 & 5.6 \\
\hline
\end{tabular}

Two participants reported that they could not use the risk assessment, and so they do not have PR. Both of these participants had a diabetes diagnosis. Of the participants who reported they "don't know" their risk: $N=1$ for colorectal cancer, who had average FSR; $N=1$ for breast cancer, who had average FSR; and $N=2$ for diabetes who both had increased FSR. ND, not diagnosed; FSR, Families SHARE risk, algorithm; PR, participants' perceived risk after receiving Families SHARE.

bers, including first-degree relatives like parents and siblings, and so they were simply "not easily available."

\section{Understanding of the Families SHARE Workbook}

Participants' self-reported understanding of the overall workbook was high $(\mathrm{M}=6.5, \mathrm{SD}=0.8)$, as was each section of the tool: the cover letter $(\mathrm{M}=6.9, \mathrm{SD}=0.4)$, the sample FHH tree $(\mathrm{M}=6.8, \mathrm{SD}=0.7)$, their own FHH tree $(\mathrm{M}=6.7, \mathrm{SD}=0.7)$, the disease factsheet and risk worksheet $(M=6.7, S D=0.7)$, and the healthy recommendations $(\mathrm{M}=6.9, \mathrm{SD}=0.2)$. Almost all participants $(94 \%)$ agreed the Families SHARE workbook helped them identify ways to reduce their or their family members' disease risk.

Although there was strong self-reported understanding, some participants had inaccurate perceptions of their disease risk after using the workbook. The accuracy of participants' disease risk perceptions was evaluated by comparing participant's risk based on the Families SHARE algorithm (FSR), with their self-reported perceived risk (PR) for each disease (see Table 3). Most participants (94.1\%) were at increased risk of at least one disease represented in the workbook based on the algorithm: $9.8 \%$ were at risk of only one disease, $62.7 \%$ were at risk of 2 diseases, and $21.6 \%$ were at risk of 3 diseases. For the 49 participants who indicated that they were able to use the risk algorithm, the proportion of nondiagnosed participants who had accurate PR was $47.5 \%$ for diabetes, $42.8 \%$ for heart disease, $82.4 \%$ for colon cancer, and $66.7 \%$ (of females) for breast cancer. Of the nondiagnosed participants who had inaccurate risk perceptions, under- estimation of their risk (i.e., increased risk based on the Families SHARE algorithm, but average perceived risk) was most common for heart disease (51.0\%), diabetes (42.5\%), and breast cancer (22.2\%) and less common for colorectal cancer (5.9\%). Among nondiagnosed participants, overestimation of their risk (i.e., increased perceived risk, but average risk based on the Families SHARE algorithm) was low for all diseases: 5.0\% for diabetes, $2.0 \%$ for heart disease, $5.9 \%$ for colorectal cancer, and $5.6 \%$ (of nondiagnosed females) for breast cancer.

Of note, accuracy here is based on consistency between participants' disease risk perceptions and the risk assessment from the Families SHARE algorithm. Since participants were asked if they were at increased risk for each disease, rather than explicitly asked what the workbook indicated as their level of risk, it is plausible that participants had accurately computed their risk using the workbook algorithm but held different beliefs about what their disease risk actually was.

The focus groups provided insights into the misalignment between some of the inaccuracies in risk perceptions, and strong self-reported understanding of the workbook. The discussions highlighted that participant's understanding of their disease risk strongly relied on how they interpreted their personalized pedigree, as well as their general understanding of disease risk in their community. And these components were more salient than the information about the level of risk (average or increased) indicated by the Families SHARE algorithm. For example, participants described drawing conclusions about their disease risk based on their personalized pedi- 
Table 4. Differences in intentions/confidence to change health behaviors from baseline to follow-up

\begin{tabular}{lcccc}
\hline Behavior & Baseline & Follow-up & $t$ value & $p$ value \\
\hline Stop smoking (smokers only) & & & & \\
All $(N=17)$ & $5.0(2.2)$ & $4.9(2.5)$ & -0.22 & 0.83 \\
Increased FSR $(N=11)$ & $5.0(2.2)$ & $4.9(2.5)$ & -0.22 & 0.83 \\
Increased PR $(N=10)$ & $5.2(1.9)$ & $5.5(2.1)$ & 0.76 & 0.47 \\
Decrease alcohol use & & & & \\
All $(N=51)$ & $0.7(2.0)$ & $0.3(1.3)$ & -1.90 & 0.06 \\
Increased FSR $(N=48)$ & $0.6(1.9)$ & $0.2(1.1)$ & -1.81 & 0.08 \\
Increased PR $(N=30)$ & $0.8(2.2)$ & $0.2(1.1)$ & -1.88 & 0.07 \\
Increase physical activity & & & & \\
All $(N=51)$ & $3.5(3.2)$ & $3.8(3.3)$ & 0.52 & 0.61 \\
Increased FSR $(N=48)$ & $3.5(3.2)$ & $3.7(3.2)$ & 0.44 & 0.66 \\
Increased PR $(N=30)$ & $3.5(3.3)$ & $3.5(3.4)$ & 0.09 & 0.93 \\
Increase fruit/vegetable & & & & \\
intake & & & & \\
All $(N=51)$ & $3.4(3.1)$ & $3.8(3.3)$ & 0.70 & 0.49 \\
Increased FSR $(N=48)$ & $3.3(3.1)$ & $3.8(3.2)$ & 1.05 & 0.30 \\
Increased PR $(N=30)$ & $3.9(3.0)$ & $4.0(3.2)$ & 0.22 & 0.83 \\
Increase fiber intake & & & & \\
All $(N=51)$ & $1.2(2.5)$ & $1.6(2.8)$ & 0.81 & 0.42 \\
Increased FSR $(N=48)$ & $1.3(2.6)$ & $1.5(2.7)$ & 0.53 & 0.30 \\
Increased PR $(N=30)$ & $1.6(2.7)$ & $1.7(2.8)$ & 0.11 & 0.91 \\
\hline
\end{tabular}

Intention/confidence: $0=$ do not intend to change the behavior, $1=$ intend to change the behavior, but not at all confident, up to $7=$ intend to change the behavior and very confident. FSR, Families SHARE risk, algorithm; PR, participants' perceived risk after receiving Families SHARE.

gree, which helped participants "see" the intergenerational patterns of disease they were already aware of:

"this is where it begins to find out who had what, and then find out what you might be..." (female, FG1)

"...because I look at the ones who are deceased and what they died from, and it's coming down the line." (female, FG1)

"It's the personalized family thing because as I saw people with names that I know I started seeing a pattern. I started to see a pattern and it was a very uncomfortable pattern... As my sister said, some of this stuff has got to be stopped for the next generation" (female, FG2)

During these discussions of disease risk, participants' understanding of the hereditary and nonhereditary risk factors emerged, including the role of "bad health habits" in their family, and inequitable social and environmental exposures within the African American community. Participants described

"I already knew [about disease risk]... I knew my mom and my aunt [had] cancer...seems like hereditary. But I'm looking around, like mostly all African Americans, you can just eat stuff and get cancer. So, I don't think it's just like in our bloodline." (female, FG1)

“...you can also have some control of changing your risks by your eating habits, and it still doesn't matter if I do change my eating habits, I can still have something I can't prevent..." (female, FG1)

\section{Intentions and Confidence to Change Health \\ Behaviors}

Changes in intentions/confidence to engage in disease risk-reducing behaviors from baseline to follow-up were assessed using paired samples $t$ tests. Results are summarized in Table 4 for the whole sample and for subsets of participants who had increased risk for any of the 4 diseases based on the Families SHARE algorithm and their reported perceived risk. Intentions to change behaviors were strongest for stopping smoking, increasing physical activity, and increasing fruit and vegetable intake; intentions to increase fiber intake or decrease alcohol use were low. There were no differences in intentions to change behavior between baseline and follow-up. There was a trend that participants' intentions to decrease alcohol use declined after the intervention, and this pattern was evident in the whole sample and those with increased disease risk.

Discussion during the focus groups suggested the Families SHARE workbook increased participants' awareness of health behaviors associated with disease risk, such as diet and physical activity. Some described changes to health behaviors they were starting to make, while others talked about changes less concretely, for example,

"I have to be mindful of my cholesterol and my salt and sugar intake (female, FG1).”

Participants typically agreed with statements that changing health behaviors was something they could control, but described the difficulty of changing habits, for example,

"we're so used to doing things a certain way it's just difficult for us to change our ways a lot of times, even when we know something is not good for us." (female, FG2)

Participants in the focus group discussed how to change health habits and some of their barriers in knowledge and resources to do so. For example, when participants were asked how to increase fiber intake, there was some need to increase understanding about how to do this. Participants reported,

"It's like my rice and oatmeal and stuff like that." (female, FG1) "Metamucil or something.... Isn't Metamucil fiber? Yeah, like take that." (female, FG1) 


\section{Participant Feedback and Recommendations}

Based on responses to an open-ended survey question, only 9 participants noted confusing aspects of the workbook. Six (6) survey respondents suggested the workbook could be improved by including more information (this suggestion came from 3 of the 9 participants that noted confusing aspects). There were recommendations for information and resources for healthy behavior change, such as resources for "becoming sober," quitting smoking, and "exercise and diet ideas." Most suggestions focused on resources for healthy eating: for example, recommendations for fruits and vegetables to eat, places to purchase fruits and vegetables, healthy recipes, and instructions on how to prepare healthy foods/healthier meals.

Discussion in the focus group elicited several themes around feedback and recommendations:

i. FHH information gathering. Participants recommended that they be given the opportunity to gather family health information before being asked to provide information about their FHH, for example, "Touch base with the person and let them know exactly what you're going to need in order to do this" (female, FG1). Related to this were discussions about how participants viewed the process of completing their personalized pedigree as a work in progress. They wanted to gather information from family members about their family history and be able to update their personalized pedigree. They suggested the Families SHARE program include additional neighborhood workshops or an online tool, where they could add information about their FHH and get an updated, printed version of their personalized pedigree. Participants mentioned wanting to update the family tree with their children's disease history and "tailoring" the tree for their children, for example,

"She [daughter] has a cholesterol history the way she eats. So, I would have her, if they had her on it, and she looked through it and seen what's going on with it, it might make her think, 'Well, yeah, I need to have this checked out for myself, and I need to have that checked out.' So, it would get her interested in it as well if they had her in it, yeah." (female, FG1)

This suggests additional supports may be needed to help users "pivot" to interpreting disease risk from the viewpoint of a child using the risk algorithm.

ii. Additional diseases and health conditions. Focus group participants recommended additional diseases and health conditions relevant to their family and community be added to the workbook. They wanted the opportunity to "add" diseases or conditions into their personalized pedigree that were relevant to them, in- cluding "alcoholism," "cirrhosis of the liver," "lung cancer," and "depression." One participant stated, "You can't add those diseases in the 'black group' without adding HIV."

iii. Additional information and resources. Participants suggested adding more information and resources about the focal diseases (e.g., "And add places where they can go look up about the diseases.") and how to adopt health behaviors. The discussion and open-ended feedback on the surveys indicated a desire for more support in developing the skills to adopt recommended health behaviors.

\section{Discussion}

The Families SHARE tool shows promise as a community-based FHH tool grounded in a family-centered, train-the-trainer perspective, to be used, understood, and valued by under-resourced African American populations. The main impact of the tool appears to be on increasing the relevance, meaning, and sense of ownership of FHH information, and in activating communication about FHH among family members, close social connections, and health-care providers. Both the quantitative and qualitative data indicated that participants used the tool to assess their own and other family members disease risk, and engagement with the tool prompted communication about FHH with family and friends, "family historians," other social contacts, and health-care providers. A goal of Families SHARE is to increase family communication about their health history to initiate communal coping $[14,15]$, and results from this pilot study indicate that these group processes were activated in a short period of time (i.e., within 6 weeks of receiving the workbook). Additionally, family and community identity (e.g., references to family members, the African American community, and the "Black group") was a common referent when discussing FHH and use of the workbook in the focus groups.

Another theme that emerged in the qualitative analyses was the value placed on feeling a sense of ownership and empowerment through the use and sharing of the workbook material. Participants saw the information as "theirs," something "they owned" and could use, and that gave them power in their interactions with health-care professions (some "ammo"). This may be meaningful in a population with a history of health inequities and medical mistrust [12].

Through their qualitative feedback, participants noted some barriers in their use of the workbook, including a 
lack of information about some family members' health history. This may be a challenge more commonly faced by low-income African American families, who have higher rates of single-parent households. In our sample, all participants had at least one child, but $20 \%$ were married or living with a spouse/partner.

Despite strong self-reported use, value, and understanding of the workbook material, we found some participants underestimated their disease risk at follow-up. This contrasts with findings from the Healthy Black Family Project (HBFP), which was successful in using FHHs to promote accurate risk perceptions for breast and colon cancer [25]. Differences in our studies were that we included material on more common diseases - diabetes and heart disease - where risk may be complex to interpret, and we worked with a population with lower education. Our community-based intervention relied on participants to interpret the material from the workbook on their own, while the HBFP utilized genetic counselors, an approach that may provide more support in interpreting FHHs but is less scalable. Analysis of the qualitative data suggests participants in our study relied heavily on the visual of their personalized pedigrees, and they may not have engaged with the risk algorithm to determine personal disease risk in the way we intended. Support may be needed for individuals in this population to utilize this component of the intervention.

The Families SHARE intervention was not successful in increasing intentions to engage in risk-reducing health behaviors in this population. This could be because participants and their families were at an early stage of the communal coping process, having received the material 6 weeks earlier. We found evidence they had started to communicate about their family health risks - the first stage of communal coping - but it may take time for families to shift into later stages of coping where they adopt and co-engage in risk-reducing behavior changes [15]. In a FHH-based education program among Mexican American families, family communication about risk and updating family histories occurred in the first few months, and it was not until 10 months after having received the material that there was stronger evidence of behavioral supports and behavior change [31]. Alternatively, providing information about health behaviors to change, with minimal information or resources about how to do this, is not a comprehensive intervention strategy for underresourced families and communities. As discussed in the focus groups, it is "difficult for us to change our ways" and people need support in "how to" change health habits. It may be the $\mathrm{FHH}$ intervention is effective at increasing perceived susceptibility but needs to be paired with other intervention components to enable behavior change in populations with significant barriers [32].

Overall, our findings indicate Families SHARE is an engaging $\mathrm{FHH}$ tool that can increase the salience of and communication about FHH among African American populations and knowledge of disease risk. The initial activation of these cognitive and social processes may support communal coping and the future adoption of risk-reducing health behaviors, particularly if they are paired with additional intervention components that address ecological barriers to behavior change and maintenance. It is likely that this tool can be further tailored to optimize its value and benefits for low-income African American populations.

\section{Acknowledgments}

The authors would like to thank Andrea Georgen, Christopher Marcum, Alicia Miggins, Amanda Ludden, Cynthia Fiorino, and BETAH Associates for their help with the study.

\section{Statement of Ethics}

The study protocol was approved by the Institutional Review Board of the National Human Genome Research Institute (NHGRI: 12-HG-0023). Informed consent to complete the study surveys was obtained from eligible participants by phone, and participants in the focus groups provided written informed consent.

\section{Conflict of Interest Statement}

The authors have no conflicts of interest to declare.

\section{Funding Sources}

This research was supported by the Division of Intramural Research Program of the National Human Genome Research Institute (ZIA HG200335, PI: Koehly).

\section{Author Contributions}

Kayla de la Haye contributed to the study design and implementation, contributed to the data analysis, and was lead writer of the paper. Calandra Whitted contributed to the data analysis and writing the paper. Laura M. Koehly is the primary investigator leading the study. She performed the data analysis and contributed to writing the paper. 


\section{References}

1 Scheuner MT, Wang SJ, Raffel LJ, Larabell SK, Rotter JI. Family history: a comprehensive genetic risk assessment method for the chronic conditions of adulthood. Am J Med Genet. 1997;71(3):315-24.

2 Lin J, Marcum CS, Wilkinson AV, Koehly LM. Developing shared appraisals of diabetes risk through family health history feedback: the case of Mexican-heritage families. Ann Behav Med. 2018;52(3):262-71.

3 Valdez R, Yoon PW, Qureshi N, Green RF, Khoury MJ. Family history in public health practice: a genomic tool for disease prevention and health promotion. Annu Rev Public Health. 2010;31:69-87.

4 Allen CG, Duquette D, Guan Y, McBride CM. Applying theory to characterize impediments to dissemination of community-facing family health history tools: a review of the literature. J Community Genet. 2020;11(2):147-59.

5 Guttmacher AE, Collins FS, Carmona RH. The family history - more important than ever. N Engl J Med. 2004;351(22):2333-6.

6 Harrison TA, Hindorff LA, Kim H, Wines RC, Bowen DJ, McGrath BB, et al. Family history of diabetes as a potential public health tool. Am J Prev Med. 2003;24(2):152-9.

7 McBride CM, Bowen D, Brody LC, Condit CM, Croyle RT, Gwinn M, et al. Future health applications of genomics: priorities for communication, behavioral, and social sciences research. Am J Prev Med. 2010;38(5):556-65.

8 Hicken B, Tucker D. Impact of genetic risk feedback: perceived risk and motivation for health protective behaviours. Psychol Health Med. 2002;7(1):25-36.

9 Ruffin MT, Nease DE, Sen A, Pace WD, Wang C, Acheson LS, et al. Effect of preventive messages tailored to family history on health behaviors: the family healthware impact trial. Ann Fam Med. 2011 Jan-Feb;9(1):3-11.

10 Koehly LM, Persky S, Spotts E, Acca G. Social and behavioral science priorities for genomic translation. Transl Behav Med. 2018;8(1): $137-43$.

11 Taylor RJ, Chatters LM, Woodward AT, Brown E. Racial and ethnic differences in extended family, friendship, fictive kin and congregational informal support networks. Fam Relat. 2013;62(4):609-24.
12 Shelton RC, Winkel G, Davis SN, Roberts N, Valdimarsdottir H, Hall SJ, et al. Validation of the group-based medical mistrust scale among urban black men. J Gen Intern Med. 2010;25(6):549-55.

13 Koehly LM, Morris BA, Skapinsky K, Goergen A, Ludden A. Evaluation of the families SHARE workbook: an educational tool outlining disease risk and healthy guidelines to reduce risk of heart disease, diabetes, breast cancer and colorectal cancer. BMC Public Health. 2015;15:1120.

14 Lewis MA, McBride CM, Pollak KI, Puleo E, Butterfield RM, Emmons KM. Understanding health behavior change among couples: an interdependence and communal coping approach. Soc Sci Med. 2006;62(6):1369-80.

15 Lyons RF, Mickelson KD, Sullivan MJL, Coyne JC. Coping as a communal process. J Soc Pers Relat. 1998;15(5):579-605.

16 Walker RJ, Strom Williams J, Egede LE. Influence of race, ethnicity and social determinants of health on diabetes outcomes. Am J Med Sci. 2016;351(4):366-73.

17 Centers for Disease Control and Prevention. CDC health disparities and inequalities report-United States. 2013.

18 Centers for Disease Control and Prevention. National diabetes statistics report. 2017.

19 Hales CM, Fryar CD, Carroll MD, Freedman DS, Aoki Y, Ogden CL. Differences in obesity prevalence by demographic characteristics and urbanization level among adults in the United States, 2013-2016. JAMA. 2018; 319(23):2419-29.

20 Mozaffarian D, Benjamin EJ, AS, Arnett DK, MJ, Cushman M, et al. Heart disease and stroke statistics: 2015 update. Circulation. 2015;131(4):e29-322.

21 Pew Research Center. Parenting in America. 2015.

22 Taylor RJ, Chatters LM, Hardison CB, Riley A. Informal social support networks and subjective well-being among African Americans. J Black Psychol. 2001;27(4):439-63.
23 Ford AF, Reddick K, Browne MC, Robins A, Thomas SB, Crouse Quinn S. Beyond the cathedral: building trust to engage the African American community in health promotion and disease prevention. Health Promot Pract. 2009;10(4):485-9.

24 Thomas SB, Quinn SC, Butler J, Fryer CS, Garza MA. Toward a fourth generation of disparities research to achieve health equity. Annu Rev Public Health. 2011;32:399.

25 Murthy VS, Garza MA, Almario DA, Vogel KJ, Grubs RE, Gettig EA, et al. Using a family history intervention to improve cancer risk perception in a black community. J Genet Couns. 2011;20(6):639-49.

26 Lin J, Marcum CS, Myers MF, Koehly LM. Racial differences in family health history knowledge of type 2 diabetes: exploring the role of interpersonal mechanisms. Transl Behav Med. 2018;8(4):540-9.

27 Kupfer SS, McCaffrey S, Kim KE. Racial and gender disparities in hereditary colorectal cancer risk assessment: the role of family history. J Cancer Educ. 2006;21(1 Suppl):S32-6.

28 Hovick SR, Yamasaki JS, Burton-Chase AM Peterson SK. Patterns of family health history communication among older African American adults. J Health Commun. 2015;20(1): 80-7.

29 Hood SM, Golembiewski EM, Sow H, Benbow K, Prather J, Robison LD, et al. Structural and contextual patterns in family health history knowledge among African American adults: a mixed-methods social network analysis study. J Soc Struct. 2019;20(3):96-118.

30 Progeny Software LLC. Progeny CLINICAL version (Version 8.0). Delray Beach (FL). 2011.

31 Lin J, Myers MF, Wilkinson AV, Koehly LM. Activating communal coping related to diabetes risk in Mexican-Heritage families. Fam Community Health. 2019;42(4):245-53.

32 Bird CE, Rieker PP. Gender and health: the effects of constrained choices and social policies. Cambridge: Cambridge University Press; 2008 